\title{
Physiological and anthropometric determinants of sport climbing performance
}

\author{
Christine M Mermier, Jeffrey M Janot, Daryl L Parker, Jacob G Swan
}

\begin{abstract}
Objective-To identify the physiological and anthropometric determinants of sport climbing performance.

Methods-Forty four climbers (24 men, 20 women) of various skill levels (self reported rating $5.6-5.13 \mathrm{c}$ on the Yosemite decimal scale) and years of experience (0.10-44 years) served as subjects. They climbed two routes on separate days to assess climbing performance. The routes (11 and $30 \mathrm{~m}$ in distance) were set on two artificial climbing walls and were designed to become progressively more difficult from start to finish. Performance was scored according to the system used in sport climbing competitions where each successive handhold increases by one in point value. Results from each route were combined for a total climbing performance score. Measured variables for each subject included anthropometric (height, weight, leg length, arm span, \% body fat), demographic (self reported climbing rating, years of climbing experience, weekly hours of training), and physiological (knee and shoulder extension, knee flexion, grip, and finger pincer strength, bent arm hang, grip endurance, hip and shoulder flexibility, and upper and lower body anaerobic power). These variables were combined into components using a principal components analysis procedure. These components were then used in a simultaneous multiple regression procedure to determine which components best explain the variance in sport rock climbing performance.
\end{abstract}

Results-The principal components analysis procedure extracted three components. These were labelled training, anthropometric, and flexibility on the basis of the measured variables that were the most influential in forming each component. The results of the multiple regression procedure indicated that the training component uniquely explained $58.9 \%$ of the total variance in climbing performance. The anthropometric and flexibility components explained $0.3 \%$ and $1.8 \%$ of the total variance in climbing performance respectively.

Conclusions-The variance in climbing performance can be explained by a component consisting of trainable variables. More importantly, the findings do not support the belief that a climber must necessarily possess specific anthropometric characteristics to excel in sport rock climbing. (Br F Sports Med 2000;34:359-366)

Keywords: rock climbing; strength; muscular endurance; training; anthropometric determinants
Research interest in rock climbing has increased since the late 1970s, in part because of increased participation in the sport. One of the first studies of the physiology of rock climbing performance was by Williams et al. ${ }^{1}$ Since then, the focus of research has shifted from outdoor rock climbing to indoor sport climbing, which has given researchers better control over extraneous variables. This shift coincides with the emergence of sport climbing as a competitive event. $^{2}$

Despite the increased research in this area, there is still some debate, as well as conflicting evidence, in the climbing literature about which physiological and anthropometric factors are important in determining climbing performance. Mermier et $a l^{\beta}$ examined the physiological responses during rock climbing and found a non-linear relation between heart rate and oxygen consumption $\left(\mathrm{VO}_{2}\right)$, which suggests that $\mathrm{Vo}_{2}$ may have a small role in determining climbing performance. Billat et $a l^{4}$ concluded that the overall percentage of maximum $\mathrm{VO}_{2}$ required is relatively small during climbing. However, in a recent study by Booth et al,,$^{5}$ moderately difficult climbing was shown to elicit a significant portion of climbing specific peak $\mathrm{VO}_{2}$ in elite climbers. Other studies have attempted to identify specific physical characteristics present in elite climbers. ${ }^{26}$ Watts et al concluded that climbing performance is best predicted by percentage body fat $(\% \mathrm{BF})$ and strength to body mass ratio in elite sport climbers. Grant et $a l^{6}$ found that elite climbers differ from recreational climbers and active non-climbers on measures of leg span, $\% \mathrm{BF}$, flexibility, and muscular strength and endurance.

It is evident that the determination of components related to climbing performance needs further investigation. The goal of this study is to improve our understanding of which components determine climbing performance by using a larger and more diverse sample within the climbing population, as well as more advanced multivariate statistical procedures than those used in previous studies. These procedures should allow us to achieve a greater understanding of the relations among components of climbing performance, which can be used by those who wish to improve their climbing ability. Therefore the purpose of this research was to determine which anthropometric and physiological components best explain the variability in climbing performance. 
Methods

SUBJECTS

Twenty four male and 20 female volunteers, aged 18-49, were recruited from the university's student body population, the local climbing gym, and the surrounding community. Before participating in the study, the subjects completed a health history questionnaire, a climbing history questionnaire, and a consent form approved by the university's human subjects review board. The climbing history questionnaire was used to obtain information about the length, frequency, and type of climbing experience (sport, traditional, ice, aid, etc), self reported ratings (defined as highest level consistently climbed), and the specific training programmes for climbing for each subject. These variables were used to quantify the training and experience of the subjects.

Subjects were excluded on the basis of previous climbing experience (fewer than five climbs) or unsuccessful completion of a screening climb rated $\sim 5.5$ on the Yosemite decimal scale (YDS). ${ }^{7}$ Subjects were also excluded on the basis of self reported preexisting medical conditions contraindicative to the study's testing regimen and/or climbing trials. Because of the maximal exertion required for the upper and lower body Wingate tests, an age limit was imposed (men $>45$ years, women $>55$ years, all subjects $<18$ years) according to the American College of Sports Medicine's Guidelines of exercise testing and prescription ${ }^{8}$.

VISITS

Subject testing was completed over a span of three visits, two at the university and one at a local climbing gym. The variables measured at visit 1 were the performance climb 1 , bent arm hang, height, weight, arm span, leg length, isokinetic leg flexion and extension strength, isokinetic shoulder extension strength, and lower body anaerobic power. Grip strength, pincer strength, grip endurance, skinfolds for body fat, hip and shoulder range of motion, and upper body anaerobic power were measured during visit 2 . Performance climb 2 was performed during visit 3 . All visits were completed within a 14 day period. The subjects were also asked to maintain their current training regimen throughout the study.

ANTHROPOMETRIC VARIABLES OF THE SUBJECTS Height was measured without shoes to the nearest $0.5 \mathrm{~cm}$ at $\mathrm{mid}$ inspiration using a stadiometer. Subjects were weighed to the nearest $0.1 \mathrm{~kg}$ in athletic apparel without shoes on a Seca digital electronic scale (Seca Corporation, Columbia, Maryland, USA). Arm span was measured with the back against a wall and the arms outstretched laterally at the height of the shoulders. Total distance from the tip of one middle finger to the tip of the other middle finger in $\mathrm{cm}$ was noted. Ape index was calculated by dividing arm span by height. Leg length was determined using a carpenter's level placed at the level of the groin while the subject was standing. Total distance in $\mathrm{cm}$ was measured from the top of the level to the ground.

Skinfold thickness was measured to the nearest $0.5 \mathrm{~mm}$ using a Lange caliper (Cambridge Scientific Industries, Columbia, Maryland USA). All measurements were taken on the right side using anatomical sites according to the Jackson and Pollock ${ }^{9}{ }^{10}$ three site equations for both men and women. These measurements were performed until two were within $10 \%$ of each other. The equations developed by $\operatorname{Siri}^{11}$ and Heyward and Stolarczyk ${ }^{12}$ were used to convert body density to $\% \mathrm{BF}$ for men and women respectively.

\section{PHYSIOLOGICAL VARIABLES OF THE SUBJECTS} Flexibility

Range of motion (ROM) was measured at the hip and shoulder and reported in degrees. ${ }^{13}$ Subjects were allowed to warm up/stretch for five minutes before measurements were taken. All measurements were taken on the right side at maximum active ROM. The larger of two measurements at each site was recorded. Hip abduction with external rotation was measured using a goniometer while the subject was seated. The goniometer was centred at the inguinal fold at the axis of rotation with the knee bent. A bubble inclinometer (Baseline, Irvington, New York, USA) was used to assess shoulder abduction, shoulder flexion, and hip flexion. Subjects were instructed to lie supine on a mat for hip flexion measurement. The inclinometer was placed on the upper border of the patella and zeroed with the leg flat on the ground. Shoulder flexion and abduction measurements were taken while subjects were standing with palms facing inward and kept in the same plane throughout the motion. The device was placed at the mid point of the biceps brachii for flexion and on the medial deltoid for abduction. The device was zeroed with the arm relaxed at the side.

Muscular strength

Each muscular strength measurement was expressed relative to body mass to control for the effect of body size. Isokinetic strength was measured during shoulder extension and leg flexion and extension using the Cybex II isokinetic system (Lumex, Ronkonkoma, New York, USA) in conjunction with the Humac 680 computer testing program (Humac 680 System; Computer Sports Medicine, Norwood, Massachusetts, USA). The Cybex system was calibrated before each trial and set at a speed of $60 \%$ s. After a warm up of three repetitions for each movement, subjects performed six maximal repetitions. The peak torque obtained for each motion was recorded.

Grip strength was measured using the dominant hand. A hand dynamometer (Jamar, Asimow Engineering, Los Angeles, California, USA) was used for all measurements and adjusted so that the middle phalanx lined up with the handle. Subjects were given three 
trials for maximum isometric grip strength. The highest reading was recorded as grip strength.

Pincer strength was determined by using a pincer dynamometer (Pinch Gauge; Samson Preston, Bolingbrook, Illinois, USA). Subjects were instructed to use only the thumb and middle digit of their dominant hand. They were allowed three attempts to achieve a maximum value. The highest value of the three attempts was recorded.

\section{Muscular endurance}

Muscular endurance was assessed by a timed bent arm hang and grip endurance. The bent arm hang was performed on a climbing hang board (Pusher, Salt Lake City, Utah, USA) using the two biggest holds (about 28 $\mathrm{cm}$ apart) on the uppermost portion of the board. The bent arm position was chosen over a full flexion (termed lock off strength) or extension position because pilot testing showed that climbers could hold these positions for extensive periods of time without the muscles of the shoulder girdle and upper arm becoming fatigued. A ladder was used to position climbers on the hang board to minimise extraneous muscular effort. Subjects were timed from the point at which $90^{\circ}$ was obtained at the elbow joint, until the point at which this angle could no longer be maintained. The researchers determined by eye when the $90^{\circ}$ position was no longer maintained during the trial.

Grip endurance of the dominant hand was measured by timing how long the subjects could maintain $50 \%$ of their maximum voluntary contraction using a hand grip dynamometer. Time measurement started when the subject obtained the target value on the dynamometer, and was stopped when the subject could no longer maintain this value after verbal encouragement. The researchers inspected the dynamometer visually throughout the trial.

\section{Anaerobic power}

Upper and lower body anaerobic power was assessed using a Monark 824E cycle ergometer (Monark, Varburg, Sweden) interfaced with a computer. Data were collected with the OptoSensor 2000 testing software package (Sports Medicine Industries, Inc, St Cloud, Minnesota, USA). Subjects were instructed to complete a five minute warm up with no resistance. The warm up required easy pedalling $(60-70 \mathrm{rpm})$ interspersed with three "sprints" at maximum rpm. Subjects then rested for about five minutes to recover from any fatigue associated with the warm up.

Anaerobic power was assessed using the previously described Wingate testing protocol. ${ }^{14}$ Workload for the lower body was set at $0.092 \mathrm{~kg} / \mathrm{kg}$ body weight for men and $0.075 \mathrm{kp} / \mathrm{kg}$ body weight for women. ${ }^{14}$ The workload for the upper body was set at $40 \%$ of the lower body workload. Little research has documented the best workload for an upper body Wingate test, and it is difficult to choose a test protocol that optimises peak power, mean power, and percentage power decline for different people. ${ }^{15}$ However, this upper body relative workload was chosen because pilot testing showed that it elicited the highest peak and mean power output. Peak power output, mean power output, and percentage power decline were recorded. Peak power and mean power output were expressed relative to body mass.

\section{Climbing trials}

Subjects were given unlimited time to warm up, stretch, and perform easy climbing before each of the climbing trials. They were allowed one opportunity to complete a screening climb ( 5.5 YDS $)$ before further testing. There was no time limit for completing any of the climbs.

Subjects attempted performance climb 1 during visit 1 and performance climb 2 during visit 3 using a belayer and rope for safety. The climbs were performed on sight, with no prior knowledge of the route and no information or encouragement given during the climb. Subjects were allowed to view the route from the ground, but not allowed to touch any holds before the start of the trial. Only one opportunity was given to complete the routes.

Performance was scored according to the system used in sport climbing competitions where each successive handhold increases in point value by one. Subjects were given a point value for the highest handhold reached. A subjective point value was then added to the point total based on how well they used their last hold. If the subject touched but did not grasp the last hold before falling, 0.1 was added to the point value. If the subject grabbed but was unable to move off the last hold before falling, 0.5 was added to the point value. If the subject grabbed the last hold and then tried to move off of it, the score for that hold was increased by 0.9 .

The performance climbing routes were designed to begin relatively easily $(\sim 5.7)$ and increase in difficulty with each handhold/move, with a minimum number of resting positions. An attempt was made to vary the nature of the movements on both routes so as not to give advantage to any given climber. The same certified competition route setter set both routes and determined the ratings, and another experienced route setter climbed the routes to confirm the ratings.

Performance climb 1 was set to start at $\sim 5.7$ YDS and progressed with increasing difficulty to $\sim 5.12$ YDS at the top. This route consisted of 21 handholds over $11 \mathrm{~m}$ of climbing. Climb 1 was set on a vertical wall, and required a traverse around a $90^{\circ}$ corner and technical moves on small holds.

Performance climb 2 was set to start at $\sim 5.7$ YDS and progressed to $\sim 5.13$ at the top. This route consisted of 42 handholds over $30 \mathrm{~m}$ of climbing. Climb 2 consisted of $\sim 10 \mathrm{~m}$ of traverse climbing on a vertical wall using large holds. The route continued to traverse around a $45^{\circ}$ corner, and became more 
Table 1 Anthropometric and demographic characteristics of the sample $(n=44)$

\begin{tabular}{|c|c|c|c|c|c|c|}
\hline & \multicolumn{3}{|c|}{$\operatorname{Men}(n=24)$} & \multicolumn{3}{|c|}{ Women $(n=20)$} \\
\hline & Mean & $S D$ & Range & Mean & $S D$ & Range \\
\hline Age (years) & 30.4 & 6.0 & $21.0-45.0$ & 32.2 & 9.2 & $18.0-49.0$ \\
\hline Weight $(\mathrm{kg})$ & $72.8^{\star}$ & 11.6 & $40.1-94.2$ & 60.1 & 5.9 & $50.2-69.9$ \\
\hline Height $(\mathrm{cm})$ & $177.4^{\star}$ & 8.8 & $163.5-193.5$ & 166.4 & 5.7 & $157.8-192.5$ \\
\hline Leg length $(\mathrm{cm})$ & $82.7^{\star}$ & 5.9 & $74.2-96.3$ & 77.3 & 4.0 & $71.0-85.0$ \\
\hline Arm span $(\mathrm{cm})$ & $185.4^{\star}$ & 9.6 & $168.0-207.0$ & 168.6 & 8.4 & $157.0-192.5$ \\
\hline Ape index & 1.0 & 0.02 & $1.00-1.08$ & 1.0 & 0.03 & $0.96-1.11$ \\
\hline Body fat $(\%)$ & $9.8^{\star}$ & 3.5 & $3.3-17.2$ & 20.7 & 4.9 & $14.1-29.6$ \\
\hline Self reported rating (YDS) & $5.10 c^{\star}$ & 1.5 & $5.8-5.13 \mathrm{~d}$ & 5.9 & 1.7 & $5.6-5.12 c$ \\
\hline Total years climbing & 7.2 & 6.1 & $.10-22$ & 7.0 & 10.7 & $0.1-44.0$ \\
\hline Training (hours/week) & $7.2^{\star}$ & 5.0 & $1.0-20.0$ & 4.1 & 3.6 & $0.0-15.0$ \\
\hline
\end{tabular}

«Significantly different from women $(\mathrm{p}<0.05)$.

overhanging with smaller sloping holds for $\sim 5 \mathrm{~m}$. The last $\sim 15 \mathrm{~m}$ of the route required the ascent of a more severe vertical overhanging section on small sloping holds. No climber in the study was able to complete climb 2 .

\section{STATISTICAL ANALYSIS}

Standard descriptive statistics (means, standard deviation, range) were used to present the characteristics of the subjects for all variables. The means of some variables differed significantly between men and women; therefore descriptive statistics for men and women are reported separately. A Pearson's productmoment correlation was used to determine the relation between climbing performance routes and climbing skill.

A principal components analysis (PCA) was used to reduce the large number of anthropometric and physiological variables to a smaller number of components. In addition, a simultaneous multiple regression procedure was used to determine the amount of variance in climbing performance that could be explained using the components derived from the PCA. An SPSS statistical package (version 8.0; SPSS, Inc, Chicago, Illinois, USA) was used. Alpha level was set at 0.05 for all analyses.

\section{Results}

PCA PROCEDURE

Tables 1 and 2 give the means, standard deviations, and ranges for the anthropometric and physiological variables. The sample exhibited a wide range of scores for all measured variables.

PCA was used to reduce the original set of variables to a smaller set that accounts for most of the variance in the initial variables and to determine which variables could be combined to best reflect underlying structures or processes related to rock climbing performance. This optimises the case to variable ratio and increases power for subsequent analyses. ${ }^{16}$ The PCA was initially performed with men and women as separate groups. However, the two analyses produced the same components, therefore data for the men and women were combined for all subsequent analyses. PCA with oblique rotation was performed initially on 25 variables for a sample of 44 men and women. The PCA was rerun with 17 variables after 12 variables were omitted because of having very low communalities or not loading on any component. ${ }^{16}$ Barlett's test of sphericity was significant $(p<0.001)$, indicating that it was reasonable to proceed with PCA even considering the small sample size.

Three components were extracted on the basis of the analysis of the scree plot, requiring 17 iterations for rotation. Oblique rotation was chosen because of a moderate correlation between components 1 and $2(r=$ $-0.312)$. Variables were well defined by the component solution, and communalities $\left(h^{2}\right)$ tended to be high. Table 3 shows loading of variables on components, communalities, and percentage of variance and cumulative variance. With a cut off of 0.40 for inclusion of a variable in interpretation of a component, ${ }^{16}$ all variables loaded on at least one component.

Table 2 Mean muscular strength, endurance, flexibility and power output characteristics of the sample $(n=44)$

\begin{tabular}{|c|c|c|c|c|c|c|}
\hline & \multicolumn{3}{|c|}{$\operatorname{Men}(n=24)$} & \multicolumn{3}{|c|}{ Women $(n=20)$} \\
\hline & Mean & $S D$ & Range & Mean & $S D$ & Range \\
\hline Knee extension (ft lbs/kg BW) & $2.09^{\star}$ & 0.24 & $1.37-2.59$ & 1.72 & 0.27 & $0.95-2.13$ \\
\hline Knee flexion (ft lbs/kg BW) & $1.40^{\star}$ & 0.20 & $1.09-1.89$ & 1.20 & 0.28 & $0.79-2.10$ \\
\hline Shoulder extension (ft lbs/kg BW) & $1.30^{\star}$ & 0.22 & $0.86-1.75$ & 0.91 & 0.13 & $0.69-1.11$ \\
\hline Bent arm hang time (seconds) & $51.80^{\star}$ & 14.62 & $19.0-90.0$ & 25.05 & 14.41 & $3.00-56.60$ \\
\hline Grip strength $(\mathrm{kg} / \mathrm{kg} \mathrm{BW})$ & $0.65^{\star}$ & 0.14 & $0.39-0.95$ & 0.49 & 0.10 & $0.35-0.65$ \\
\hline Pincer strength (kg/kg BW) & 0.14 & 0.03 & $0.10-0.21$ & 0.12 & 0.02 & $0.06-0.16$ \\
\hline Grip endurance (seconds) & 79.69 & 37.17 & $19.50-174.0$ & 79.78 & 29.82 & $34.00-174.00$ \\
\hline Hip flexion (degrees) & 137.2 & 15.9 & $100.0-165.0$ & 142.3 & 13.3 & $104.0-165.0$ \\
\hline Hip abduction (degrees) & 89.4 & 20.2 & $62.0-133.0$ & 92.0 & 21.2 & $63.0-140.0$ \\
\hline Shoulder flexion (degrees) & 180.8 & 17.8 & $135.0-208.0$ & 185.7 & 15.8 & $152.0-210.0$ \\
\hline Shoulder abduction (degrees) & $146.8^{\star}$ & 35.2 & $74.0-195.0$ & 170.9 & 17.6 & $145.0-199.0$ \\
\hline Wingate lower body peak (W) & $1230.71^{\star}$ & 229.44 & $575.0-1620.0$ & 728.85 & 115.57 & $468.0-929.0$ \\
\hline Wingate lower body peak (W/kg BW) & $16.87^{\star}$ & 1.56 & $13.84-19.80$ & 12.15 & 1.57 & $8.24-14.90$ \\
\hline Wingate lower body mean (W) & $639.04^{\star}$ & 103.0 & $361.0-873.0$ & 407.1 & 50.0 & $319.0-524.0$ \\
\hline Wingate lower body decline (\%) & 64.58 & 7.11 & $49.0-75.0$ & 59.4 & 12.09 & $19.0-77.0$ \\
\hline Wingate upper body peak (W) & $494.42^{\star}$ & 120.87 & $199.0-766.0$ & 289.0 & 45.11 & $207.0-389.0$ \\
\hline Wingate upper body peak (W/kg BW) & $6.80^{\star}$ & 0.85 & $4.96-8.20$ & 4.80 & 0.60 & $3.38-5.80$ \\
\hline Wingate upper body mean (W) & $328.37^{\star}$ & 61.92 & $158.0-420.0$ & 194.25 & 23.55 & $128.0-134.0$ \\
\hline Wingate upper body decline (\%) & 54.96 & 9.39 & $37.0-77.0$ & 53.0 & 11.44 & $10.0-64.0$ \\
\hline
\end{tabular}

*Significantly different from women $(\mathrm{p}<0.05)$.

$\mathrm{ft}$ lbs/kg BW, foot pounds per kilogram of body weight; W/kg BW, Watts per kilogram of body weight; $\mathrm{kg} / \mathrm{kg}$ BW, kilograms of force per kilogram of body weight. 
Table 3 Component loadings: pattern $(P)$ and structure $(S)$, communalities $\left(h^{2}\right), \%$ of variance, and cumulative $\%$ of variance for principal component analysis on climbing variables

\begin{tabular}{|c|c|c|c|c|}
\hline Items & Component $_{1} P / S$ & Component $_{2} P / S$ & Component $_{3} P / S$ & $h^{2}$ \\
\hline Weight & & $-0.82 /-0.84$ & & 0.81 \\
\hline Height & & $-1.00 /-0.96$ & & 0.94 \\
\hline Leg length & & $-0.94 /-0.86$ & & 0.81 \\
\hline Arm span & $/ 0.38$ & $-0.87 /-0.94$ & & 0.96 \\
\hline Body fat & $-0.85 /-0.89$ & $/ 0.43$ & & 0.90 \\
\hline Ape index & & $/-0.45$ & & 0.78 \\
\hline Hip flexion & & & $0.75 / 0.75$ & 0.59 \\
\hline Hip abduction & & & $-0.75 /-0.71$ & 0.77 \\
\hline Hang time & $0.91 / 0.90$ & & & 0.84 \\
\hline Lower body power & $0.53 / 0.68$ & $/-0.57$ & & 0.81 \\
\hline Upper body power & $/ 0.59$ & $-0.50 /-0.72$ & & 0.79 \\
\hline Climbing rating & $0.74 / 0.70$ & & & 0.70 \\
\hline Experience & & & $0.64 / 0.67$ & 0.61 \\
\hline Knee extension & $0.51 / 0.59$ & & & 0.60 \\
\hline Knee flexion & $0.71 / 0.71$ & & & 0.64 \\
\hline Shoulder extension & $0.89 / 0.91$ & & & 0.85 \\
\hline Grip strength & $0.81 / 0.79$ & & & 0.70 \\
\hline$\%$ of variance & 39.06 & 15.35 & 10.36 & \\
\hline Cumulative \% & 39.06 & 54.41 & 64.77 & \\
\hline
\end{tabular}

Component labels: Component $_{1}=$ training, Component $_{2}=$ anthropometric, Component $_{3}=$ flexibility.

Table 4 Zero order correlations among the predictor components and climbing performance $(n=44)$

\begin{tabular}{lllll}
\hline Component & 1 & 2 & 3 & 4 \\
\hline 1. Climbing performance & - & $0.798^{\star}$ & -0.195 & 0.168 \\
2. Training & & - & $-0.312^{\star}$ & 0.037 \\
3. Anthropometric & & & - & 0.029 \\
4. Flexibility & & & - \\
\hline${ }^{\star} \mathrm{p}<0.05$. & & & &
\end{tabular}

Labelling of components can be highly subjective. The components are operational representatives of the construct implicit in the label and do not "explain" the variables. Suggested interpretative labels for each component were derived from the variables that were the most influential in forming the components. Component 1 was named "training" because of strong loadings of variables deemed to be trainable such as knee and shoulder strength, grip strength, upper and lower body power, hang time, $\% \mathrm{BF}$, and self reported sport climbing rating. Component 2 was named "anthropometric" as weight, height, leg length, arm span, and ape index were the most influential variables. Component 3 was labelled "flexibility" because of the strong influence of both hip ROM variables in forming the component, although climbing experience was also included.

MULTIPLE REGRESSION PROCEDURE

The criterion measure for this study was represented by a total climbing performance score over two climbing routes. A Pearson's productmoment correlation was calculated to determine if the scores from the two routes could be combined into a total performance score. This value $(r=0.81)$ indicated that the two routes were measuring similar climbing abilities; subsequently, one total score was used as the dependent variable. The total possible score

Table 5 Set of all predictor components for climbing performance $(n=44)$

\begin{tabular}{llllll}
\hline Component & $B$ & $\beta$ & Part $r^{2}$ & $t$ & $p$ \\
\hline Training & 9.967 & 0.809 & 0.589 & 8.31 & 0.001 \\
Anthropometric & 0.660 & 0.054 & 0.003 & 0.550 & 0.585 \\
Flexibility & 1.679 & 0.136 & 0.018 & 1.47 & 0.149 \\
\hline
\end{tabular}

was 64 . The mean (SD) climbing performance score was $18.96(6.4)$ (range 11.2-36.4) and 30.62 (13.6) (range 11.0-59.6) for women and men respectively.

Table 4 presents the zero order correlations among the predictor components and climbing performance for the total sample. Inspection of the correlation matrix showed that only the training component and climbing performance, as well as the training and anthropometric components, show a significant relation to one another. It is interesting to note that the training component shows a high positive relation to the criterion measure, climbing performance. This suggests that higher climbing performance scores are associated with a higher degree of training.

A simultaneous multiple regression procedure was used to determine which components were significant predictors of climbing performance in the sample. For this particular regression procedure, all components were entered concurrently to evaluate their contributions to the overall regression model. When applied simultaneously using all predictor components, the regression procedure resulted in an $R^{2}$ of 0.658 , an adjusted $R^{2}$ of 0.633 , and a standard error of the estimate of 7.46. A Durbin-Watson test was also calculated to evaluate if the independence of error assumption in the regression model was met. The value was 2.12 , which indicated that this assumption was met.

Table 5 presents the predictor components from this regression procedure for the sample. A tolerance diagnostic of multicollinearity was calculated for each predictor component to evaluate the possible presence of multicollinearity among the components. A tolerance of less than 0.10 would indicate the presence of significant multicollinearity problems. The range of tolerance for the components was from 0.901 to 0.997 , indicating the absence of significant multicollinearity problems among the components.

The standardised $\beta$ coefficients indicate the magnitude of unique contribution that each component makes to maximally predicting the criterion measure in the regression model. Only one component makes a significant contribution to the prediction model according to the $t$ values for each $\beta$ coefficient (table 5). The training component has the largest $\beta$ coefficient, meaning that it makes the largest unique contribution to the overall regression model, followed by the flexibility and anthropometric components.

The squared semi-partial correlations $\left(\right.$ part $r^{2}$ ) for the components show that about $58.9 \%, 0.3 \%$, and $1.8 \%$ of the variance in climbing performance can be uniquely explained by training, anthropometric, and flexibility respectively (table 5). The total part $r^{2}$ value is 0.610 , indicating that $61.0 \%$ of the variance in climbing performance can be accounted for by combining the unique variance explained by the three predictor components. This value can be subtracted from the $R^{2}$ value to obtain the percentage of 
explained variance that is due to overlapping of the predictor variables or multicollinearity of predictor variables. This value was calculated to be about 0.048 , meaning that $4.8 \%$ of the total explained variance was due to overlapping of the predictor variables. This relatively small amount of multicollinearity among the predictor components gives greater justification to the interpretability of the regression model results.

\section{Discussion}

Sport climbing has grown tremendously in popularity in the past few years, and standards of difficulty have continued to rise along with the number of competitions. Climbers of all abilities who are interested in improving would benefit from research into determinants of sport climbing performance. Much of the scientific literature on climbing focuses on climbing injuries and their prevention. A few recent studies have examined physiological and anthropometric variables related to climbing. ${ }^{2-61718}$ This study attempts to describe which variables explain sport climbing performance in a heterogeneous sample of climbers.

A number of recent studies contain descriptive data on elite or experienced climbers. ${ }^{2-6}$ In 1993, Watts et al compiled anthropometric data on 21 men and 18 women semifinalists at a World Cup sport climbing competition. In general, the elite climbers were characterised as being small in stature, with low $\% \mathrm{BF}$, high grip strength, and high grip strength to body mass ratio. Grant et $a l^{6}$ compared various anthropometric and physical variables of elite and recreational male climbers. Elite climbers were found to have greater upper body endurance (bent-arm hang time and pull ups), finger strength, and hip flexibility as measured by the sit and reach test.

In this study, male and female climbers tended to be similar in stature to those in the study by Watts et al. ${ }^{2}$ Also, the elite climbers in the study by Watts et $a l^{2}$ had, on average, lower body mass (66.6 $v 72.8 \mathrm{~kg}$ for men; $51.5 v 60.1$ $\mathrm{kg}$ for women), less body fat $(4.7 \% v 9.8 \%$ for men; $10.7 \%$ v $20.7 \%$ for women), greater relative grip strength $(0.78 v 0.65$ for men; $0.66 v$ 0.49 for women), and more climbing experience (11.2 $v 7.3$ years for men; $8.8 v 7.0$ years for women) than the climbers in this study. Our male climbers also compared favourably with the elite climbers in the study by Grant et $a l^{6}$ with respect to $\% \mathrm{BF}(9.8 \%$ v $14.0 \%)$ and bent arm hang time (51.8 $v 53.1$ seconds). It is important to note that the subjects in our study are more diverse as a sample with respect to climbing ability than the samples in the other studies cited. A diverse sample was selected to enhance external validity to lend greater generalisability of the results to climbers of various abilities.

Twelve variables did not load on any component in this analysis. These included age, shoulder flexibility, finger pincer strength, grip endurance, hours of training a week, and absolute measurement of peak, mean, and decrease in power for upper and lower body Wingate tests. In PCA, variables with low correlations with the important components and with low squared multiple correlation with all other variables are considered outliers. In our opinion, the fact that these variables were not extracted in the PCA does not mean that they are not important to climbing performance, rather that they were unrelated to other variables in the solution for this model. The usefulness of these variables needs to be clarified by future research.

The PCA extracted a three component model to represent variables related to sport rock climbing performance. The component (training) that captured the largest amount of cumulative variance in the PCA model $(\sim 39 \%)$ included variables that are influenced by training such as strength, power, $\% \mathrm{BF}$, and climbing skill (self reported rating). The second component included anthropometric measures such as height, weight, arm span, leg length, and ape index, and the third component included hip flexibility along with years experience, explaining about $15 \%$ and $10 \%$ of the cumulative variance for the model respectively. The importance of these findings lies in the use of this statistical method because it shows that success in sport climbing is related to the interaction of multiple variables rather than a limited number of variables as previously identified. ${ }^{2}{ }^{6}$ The relative importance of the first component underscores the need for successful training programmes to include workouts that emphasise the development of muscular strength, endurance and power, as well as climbing specific skills.

According to the results of the PCA, the three components that were extracted from the original set of variables are training, anthropometric, and flexibility. Previous research that focused on identifying variables that explain climbing ability included some variables that are associated with these components. $^{26}$ However, in our study, the training component was the only significant predictor of climbing performance, thereby reducing the importance of the anthropometric and flexibility components to overall climbing ability.

One of the most important findings of this study is that climbing ability can be significantly explained by the training component. Many climbers believe that climbing success depends on certain characteristics that are considered to be largely untrainable, such as small body stature, a positive ape index, and a specific body somatotype for climbing. It is evident from previous research that elite climbers possess similar anthropometric and physiological characteristics. Watts et al identified small to moderate stature and very low $\% \mathrm{BF}$ as being characteristics shared by elite climbers. However, when these variables, along with other anthropometric and physiological variables, were entered into a multiple regression model, only $\% \mathrm{BF}$ and grip strength to body mass ratio were considered to be significant predictors of climbing ability. These two predictor variables can be improved 
through specific modes of training, which thereby would improve climbing performance. The results from Watts et $a l^{2}$ show that, although there is a tendency among elite climbers to share certain anthropometric characteristics, they are not necessarily required to attain elite levels of climbing performance. Given the results of Watts et al, ${ }^{2}$ trainable variables are most important to climbing performance in elite climbers.

Grant et $a l^{6}$ suggested that improvements in climbing ability can be made through training to increase shoulder endurance, finger pincer strength, and hip flexibility. In our study, hip flexibility measurements contributed significantly to the third component, but were not associated with the first component (training). This is not to say that flexibility cannot be improved through training, but that the flexibility component was not a significant determinant of climbing performance in this study. These results appear to be counterintuitive especially with regard to the physical demands of sport climbing. The fact that a single flexibility component was formed using the original set of variables in this study indicates the importance of hip joint ROM to sport climbing performance.

It should also be stated that about $66 \%$ of the total variance in climbing performance was explained by the current model, leaving $34 \%$ of the variance explainable by other factors. However, considering similar attempts made by previous authors, ${ }^{2}$ the amount of explained variance in our study is relatively large. This would leave other factors, such as problem solving skills, psychological factors, and climbing specific balance, which were not included in this study, as possible predictors of climbing ability. Further investigation of these factors is warranted.

In addition, it is believed that route familiarity improves climbing performance. ${ }^{19}$ The application of skill in repeated attempts at completing the same route could have favoured less experienced climbers. In this study, this was controlled for by allowing only one attempt at each route. However, in the non-competitive setting, repeated attempts at the same route are common practice. Therefore further study is necessary to determine whether allowing unlimited practice on the routes before testing would affect the results by possibly lowering the amount of unexplained variance and increasing generalisability to common climbing practice. Another potential limitation of this study is the low generalisability of the findings to other types of climbing, such as ice, aid, or traditional climbing. Although attempts were made to vary the type of movements and holds when the routes were set, it was impossible to eliminate completely the gain or loss of advantage for any given climber. However, the power of this study lies in the specific application of the findings to sport rock climbing.

In conclusion, the results of this study show that a large portion of the variance in climbing performance can be explained by a component consisting of trainable variables. More importantly, these findings do not support the belief that a climber must possess specific anthropometric characteristics to be successful in the sport of climbing. Thus, engaging in a training programme to increase muscular strength, power, and endurance is more important than flexibility and anthropometric measurements for determining climbing performance in climbers of all abilities.

1 Williams E, Taggert P, Carruthers M. Rock climbing: observations on heart rate and plasma catecholamines and the influence of oxprenolol. Br f Sports Med 1978;12: $125-8$.

2 Watts P, Martin D, Durtschi S. Anthropometric profiles of elite male and female competitive sport rock climbers. $\mathcal{f}$ elite male and female compet
Sports Sci 1993;11:113-17.

3 Mermier C, Robergs R, McMinn S, et al. Energy expenditure and physiological responses during indoor rock climbing. Br $\mathcal{F}$ Sports Med 1997;31:224-8.

4 Billat V, Palleja P, Charlaix T, et al. Energy specificity of rock climbing and aerobic capacity in competitive sport rock climbers. F Sports Med Phys Fitness 1995;35:20-4.

5 Booth J, Marino F, Hill C, et al. Energy costs of sport rock climbing in elite performers. Br F Sports Med 1999;33:1418.

6 Grant S, Hynes V, Whittaker A, et al. Anthropometric, strength, endurance, and flexibility characteristics of elite and recreational climbers. $\mathcal{F}$ Sports Sci 1996;14:301-9.

7 Graydon D, ed. Mountaineering: the freedom of the hills. 5th ed. Seattle: The Mountaineers, 1992.

8 Kenney W, ed. ACSM's guidelines for exercise testing and prescription. 5th ed. Media, PA: Williams \& Wilkins, 1995.

9 Jackson A, Pollock M. Generalized equations for predicting body density of men. Br F Nutr 1978;40:497-504.

10 Jackson A, Pollock $M$. Generalized equations for predicting body density of women. Med Sci Sports Exerc 1980;12:175-

11 Siri W. Body composition from fluid space and density. In: Brozek J, Hanschel A, eds. Techniques for measuring body
composition. Washington, DC: National Academy of Scicomposition.

12 Heyward V, Stolarczyk L. Applied body composition assessment. 1st ed. Champaign, IL: Human Kinetics, 1996

13 Heyward V. Advanced fitness assessment and exercise prescription. 3rd ed. Champaign, IL: Human Kinetics, 1998.

14 Bar-Or O. The Wingate anaerobic test-an update on methodology, reliability and validity. Sports Med 1987;4:381-94.

15 Inbar O, Bar-Or O, Skinner J. The Wingate anaerobic test. 1st ed. Champaign, IL: Human Kinetics, 1996.

16 Tabachnick B, Fidell L. Using multivariate statistics. 3rd ed. Northridge, CA: HarperCollins College Publishers, 1996.

17 Watts P, Newbury V, Sulentic J. Acute changes in handgrip strength, endurance, and blood lactate with sustained sport rock climbing. I Sports Med Phys Fitness 1996;36: $255-60$.

18 Ferguson R, Brown M. Arterial blood pressure and forearm vascular conductance responses to sustained and rhythmic sometric exercise and arterial occlusion in trained rock climbers and untrained sedentary subjects. Eur $\mathcal{F} A p p l$ Physiol 1997;76:174-80.

19 Birkett B. Techniques in modern rock and ice climbing. London: A N C Black, 1988.

\section{Take home message}

Although it is traditionally thought that physical attributes such as height, arm length, and body weight are important in climbing performance, the variables found to explain most variance in sport climbing ability are trainable. Research to determine the most effective training programmes for sport rock climbing is still required. However, muscular strength, power, and endurance specific to sport climbing should be included in the training regimen. 


\section{Commentary}

This paper contributes some new information which builds on previous published work toward the development of an athlete model for sport climbing performance. The study includes assessment of variables that have not been previously reported and uses a principal components analysis procedure to present a different perspective of analysis. The results may have limited application, however, except to discriminate between climbers of a wide range of ability. The concept of diversity within subjects for this type of study is well illustrated in an article by Sjodin and Svedenhag, ${ }^{1}$ in which the authors illustrate that $\mathrm{VO}_{2}$ max correlates well with mean marathon running velocity for runners with velocities ranging from 3.0 to $5.4 \mathrm{~m} / \mathrm{s}(r=0.78)$. However, the correlation suffers when the sample is reduced to only "fast" runners with velocities between 4.65 and $5.40 \mathrm{~m} / \mathrm{s}(r=0.01)$. Thus the application of interpretations may be limited to samples of diverse climbing ability. Still, this work has identified possible factors that may now be studied to determine how training induced changes are reflected in changes in performance.

P WATTS

Department of Health, Physical Education and Recreation, 104 Presque Isle Avenue, Marquette, MI 49855, USA

1 Sjodin B, Svedenhag J. Applied physiology of marathon running. Sports Med 1985;2:83-99.

\section{Invitation to Delegates}

6th European Forum on Quality Improvement in Health Care Thursday 29-Saturday 31 March 2001, Bologna, Italy

For full information, contact BMA/BMJ Conference Unit, BMA House, Tavistock Square, London WC1H 9JP UK. Tel +44 (0) 207383 6409; fax: +44 (0) 207383 6869; email: quality@bma.org.uk

Bookmark our website for full information: www.quality.bmjpg.com 